\title{
Distribuição Espacial da Placa Vulnerável na Árvore Coronária: Avaliação Angiográfica de uma Coorte Consecutiva e Não-Selecionada de Pacientes Submetidos a Angioplastia Primária
}

José Fábio Almiro da Silva', Daniel Chamiéé, José Ribamar Costa Jr. ${ }^{1}$, Alexandre Abizaid ${ }^{1}$, Fausto Feres ${ }^{1}$, Francisco Carleial Feijó de Sá1, Raphael Lanza e Passos ${ }^{1}$, Luiz Alberto Mattos ${ }^{1}$, Rodolfo Staico ${ }^{1}$, Áurea J. Chaves ${ }^{1}$, Amanda G. M. R. Sousa' ${ }^{1}$, J. Eduardo Sousa ${ }^{1}$

\section{RESUMO}

Fundamentos: A gênese do infarto agudo do miocárdio (IAM) está intimamente relacionada à ruptura de "placas vulneráveis". Estudos anatomopatológicos sugerem uma distribuição não-homogênea dessas placas ao longo da árvore coronária. Neste estudo, visamos a estabelecer as "zonas vulneráveis", no sentido de obter um mapa do risco de maior probabilidade de ocorrência de IAM. Método: Neste estudo, foram analisados 82 pacientes consecutivos tratados com angioplastia primária em um centro terciário de referência, no período de janeiro a setembro de 2007. Somente foram incluídos pacientes com IAM com elevação do segmento ST, com fluxo coronário TIMI 0/1. Após definir o segmento de oclusão, utilizando a segmentação coronária do Coronary Artery Surgery Study (CASS), mensuramos a distância do ponto de oclusão do vaso culpado até seu óstio, com software dedicado (CMS-Medis). O modelo de regressão de Poisson foi utilizado para determinar o risco de IAM, baseado na distância da oclusão a partir do óstio coronário. Resultados: A média das idades foi de 60 anos e $73,1 \%$ dos pacientes eram homens. A artéria descendente anterior foi o vaso culpado mais prevalente $(46,3 \%)$. O diâmetro médio de referência do vaso foi de 2,8 \pm 0,6 mm. Para cada segmento de $10 \mathrm{~mm}$ a partir do óstio do vaso-alvo, foi observada, pela regressão de Poisson, redução de $36,9 \%$ no risco de oclusão aguda do vaso. Nesta coorte notou-se distribuição nãohomogênea das placas vulneráveis, com maior prevalência nos segmentos proximais dos vasos epicárdicos. Conclusões: Os achados desta análise in vivo estão em concordância com os estudos anatomopatológicos prévios. Identificação das "zonas vulneráveis" na árvore coronária

\section{ABSTRACT}

Spatial Distribution of Vulnerable Plaques in the Coronary Tree: an Angiographic Evaluation of a Consecutive, Non-selected Cohort Treated with Primary Angioplasty

Background: The genesis of acute myocardial infarction (AMI) seems to be closely correlated with the rupture of vulnerable plaques. Anatomical-pathological evaluations have suggested that there is a non-homogeneous distribution of these unstable plaques along the coronary arteries. We sought to establish the "vulnerable zones" in order to obtain a risk map for AMI. Methods: Eighty-two consecutive patients treated with primary angioplasty in a reference tertiary center, from January to September 2007, were enrolled in this analysis. Only ST elevation AMI patients with documented TIMI flow 0/1 were included. After defining the occluded segment using the Coronary Artery Surgery Study (CASS) classification, we measured the distance from the occlusion point in the culprit vessel to ostium using dedicated software (CMS-Medis). Poisson's regression model was used to determine the risk of AMI based on the distance between the occlusion and the coronary ostium. Results: Mean age was 60 years and most patients were men $(73.1 \%)$. LAD was the culprit vessel in most cases (46.3\%). Mean reference vessel diameter was $2.8 \pm 0.6 \mathrm{~mm}$. For each $10 \mathrm{~mm}$ segment distal from the target vessel ostium, a $36.9 \%$ reduction in the chance of acute closure of the vessel was observed by the Poisson's regression model. In this cohort there was a non-homogeneous distribution of vulnerable plaques, with higher prevalence in the proximal segments of epicardic vessels. Conclusions:

1 Instituto Dante Pazzanese de Cardiologia - São Paulo, SP, Brasil. Correspondência: José Ribamar Costa Jr. Instituto Dante Pazzanese de Cardiologia. Av. Dr. Dante Pazzanese, 500 - 14ㅇandar Ibirapuera - São Paulo, SP, Brasil - CEP 04012-180

E-mail: rmvcosta@uol.com.br

Recebido em: 2/3/2009 • Aceito em: 5/5/2009 
Silva JFA, et al. Distribuição Espacial da Placa Vulnerável na Árvore Coronária: Avaliação Angiográfica de uma Coorte Consecutiva e Não-Selecionada de Pacientes Submetidos a Angioplastia Primária. Rev Bras Cardiol Invas. 2009;17(2):209-13.

pode ajudar a selecionar dispositivos diagnósticos e de prevenção/tratamento das placas vulneráveis.

DESCRITORES: Infarto do miocárdio. Angioplastia transluminal percutânea coronária. Ruptura espontânea/fisiopatologia. Angiografia coronária.

A oclusão trombótica persistente de uma placa aterosclerótica em uma artéria coronária epicárdica é considerada a principal causa na gênese do infarto agudo do miocárdio com elevação do segmento $\mathrm{ST}^{1,2}$. Erosão ou mesmo franca ruptura dessas placas desencadeiam uma cascata patológica, com adesão fibrinoplaquetária, resultando em formação de trombo oclusivo ${ }^{3,4}$. Placas ateroscleróticas com capa fibrosa fina, conteúdo lipídico abundante e grande atividade inflamatória, conhecidas como placas vulneráveis, são as mais propensas a ruptura ${ }^{5-7}$.

Na tentativa de entender, tratar e prevenir a ocorrência das síndromes coronárias agudas, a identificação dessas placas e a adoção de medidas que promovam sua estabilização têm papel fundamental.

Baseados na teoria de que a ocorrência das placas vulneráveis tem distribuição não-uniforme ao longo da extensão do vaso coronário ${ }^{7-9}$, a maior compreensão dos locais onde elas são mais prevalentes pode ajudar na decisão de se promover modificação direta ou mesmo selamento dessas placas por meio de intervenção coronária percutânea ${ }^{10}$.

Angiografia coronária, ultrassonografia intracoronária e angioscopia têm sido utilizadas no cenário clínico da cardiologia intervencionista, com várias limitações na identificação dessas placas. Crescente esforço tem sido dispensado no desenvolvimento de novas técnicas diagnósticas, com resultados animadores.

Com o objetivo de desenvolver um modelo simples de identificação dessas placas propensas a ruptura, criamos um mapa angiográfico que identifica os segmentos da árvore coronária, em que a prevalência dessas placas é maior.

\section{MÉTODO}

\section{Seleção de pacientes}

No período de janeiro a setembro de 2007, selecionamos, de forma consecutiva, todos os pacientes que se apresentaram no Instituto Dante Pazzanese de Cardiologia, em São Paulo, com infarto agudo do miocárdio com elevação do segmento ST, encaminhados para realização de cinecoronariografia imediata seguida de angioplastia primária e que tinham presença de fluxo coronário TIMI 0 ou 1. Pacientes com menos de
The findings of this in vivo analysis are in agreement with previous anatomical-pathological studies. Identification of "vulnerable zones" in the coronary tree may help to select devices for diagnostic purposes and for the prevention/ treatment of vulnerable plaques.

DESCRIPTORS: Myocardial infarction. Angioplasty, transluminal, percutaneous coronary. Rupture, spontaneous/physiopathology. Coronary angiography.

18 anos de idade foram excluídos da análise. Excluímos, ainda, indivíduos submetidos previamente a intervenção coronária percutânea e/ou cirurgia de revascularização do miocárdio.

Infarto agudo do miocárdio com elevação do segmento ST foi definido como presença de pelo menos dois dos seguintes critérios: (1) dor torácica tipicamente anginosa; (2) supradesnivelamento mantido do segmento ST maior que $1 \mathrm{~mm}$ em duas derivações contíguas ou maior que $2 \mathrm{~mm}$ nas derivações $\vee 1$ a V3; e (3) elevação dos marcadores de necrose miocárdica (troponina) ${ }^{11}$.

\section{Análise angiográfica}

As angiografias coronárias foram analisadas em formato digital, off-line, em laboratório de análise de dados, por dois operadores independentes, utilizando-se um programa de detecção automática de bordas (QCA-CMS, Medis Medical Imaging System - Nuenen, Holanda).

Utilizamos o mapa arterial coronário do Coronary Artery Surgery Study (CASS) ${ }^{12}$ para segmentação do vaso.

A ponta do cateter-guia, totalmente preenchida por contraste, foi utilizada para calibração.

Análise quantitativa consistiu da mensuração do diâmetro de referência do vaso e da distância do ponto de oclusão até o óstio coronário, utilizando-se projeções angiográficas que melhor expusessem o segmento-alvo, com o menor grau de encurtamento.

Na análise qualitativa, classificamos o fluxo coronário de acordo com as definições do Thrombolysis In Myocardial Infarction (TIMI) ${ }^{13}$. Nos casos de fluxo coronário TIMI 0, a distância da lesão foi definida como a distância do óstio do vaso culpado até o sítio da trombose. E nos casos de fluxo coronário TIMI 1, a distância era medida do óstio coronário até o ponto do diâmetro luminal mínimo dentro da lesão culpada.

\section{Análise estatística}

As variáveis categóricas foram expressas como frequências absolutas e porcentuais e comparadas por meio dos testes exato de Fisher e de qui-quadrado. As variáveis contínuas foram apresentadas como média \pm desvio padrão e comparadas pelo teste $t$ de Student. Valores de $\mathrm{P}$ menores que 0,05 foram considerados significantes. 
Além disso, construímos um modelo de regressão de Poisson para estimar o risco de oclusão coronária como uma função da distância a partir do óstio coronário, confirmado pelo coeficiente de correlação de Spearman.

\section{RESULTADOS}

No período mencionado, 82 pacientes foram identificados e correspondem à população analisada. A média das idades foi de 60 anos, 73,2\% eram do sexo masculino e diabetes melito estava presente em 34,1\% dos casos. Os vasos mais acometidos foram a artéria descendente anterior (46,3\% dos casos) e a artéria coronária direita $(40,2 \%)$. A Tabela 1 apresenta as características clínicas da população estudada, bem como a identificação dos vasos acometidos.

A análise de regressão de Poisson demonstrou que para cada $10 \mathrm{~mm}$ em que a placa se distancia do óstio coronário há redução significativa de 36,9\% no risco de oclusão aguda do vaso. A Figura 1 apresenta um gráfico de distribuição das oclusões coronárias agudas como uma função da distância do óstio coronário. O coeficiente de correlação de Spearman foi de $-0,9478$ ( $\mathrm{P}<0,0001)$, indicando, em conjunto com o gráfico de envelope (Figura 2), que o modelo de regressão foi bem ajustado, e confirma a existência de forte correlação negativa entre a distância do óstio coronário e a ocorrência de eventos.

\section{DISCUSSÃO}

O presente trabalho avaliou a distribuição de tromboses oclusivas ao longo do vaso coronário e identificou um padrão não-uniforme de apresentação des- sas oclusões, que tendem a se concentrar nos segmentos proximais dos vasos coronários epicárdicos.

Infarto agudo do miocárdio é uma das principais causas de mortalidade e incapacidade em todo o mundo ${ }^{11}$. Estima-se uma prevalência de 500 mil casos de infarto agudo do miocárdio com elevação do segmento ST por ano nos Estados Unidos ${ }^{14}$.

A principal causa é a trombose oclusiva do vaso coronário epicárdico ${ }^{1,2}$, na maioria das vezes (cerca de $60 \%$ a $75 \%$ ) causada pela ruptura de uma placa aterosclerótica com características de instabilidade ${ }^{15,16}$. Estudos anatomopatológicos têm demonstrado que essas placas mais propensas a ruptura, conhecidas por "placas vulneráveis ${ }^{\prime 17}$, possuem alguns aspectos característicos: 1) remodelamento vascular positivo; 2) capa fibrosa fina $(<65 \mu \mathrm{m}) ; 3)$ grande lago lipídico/núcleo necrótico, geralmente contendo hemorragia e/ou calcificação; 4) calcificação com padrão difuso ou salpicado; 5) abundante rede de vasa vasorum neointimal; e 6) presença de células inflamatórias, como macrófagos ativados e linfócitos $T$, na proximidade da capa fibrosa $a^{5,7,16-20}$.

Uma vez que muitos desses determinantes de vulnerabilidade da placa são anormalidades estruturais, técnicas de imagem com alta resolução podem se tornar métodos promissores na detecção das placas vulneráveis ${ }^{21}$. As técnicas angiográficas utilizadas na rotina, embora possam identificar algumas características de ruptura da placa (como ulceração, visualização de flap intimal, bordos irregulares, presença de trombo e/ou aneurisma) ${ }^{22}$, são incapazes de identificar as lesões que têm alto risco para ruptura ${ }^{23}$.

Modalidades de imagem não-invasivas, como tomografia computadorizada ${ }^{24,25}$, ressonância magnéti-

TABELA 1

Características clínicas e identificação do vaso culpado

\begin{tabular}{|c|c|}
\hline Características & Valores $(n=82)$ \\
\hline Idade, média \pm desvio padrão & $60 \pm 11,9$ \\
\hline Sexo masculino, $\mathrm{n}(\%)$ & $60(73,2)$ \\
\hline Hipertensão arterial sistêmica, n (\%) & $58(70,7)$ \\
\hline Diabetes melito, n (\%) & $28(34,1)$ \\
\hline Dislipidemia, n (\%) & $45(54,9)$ \\
\hline Tabagismo atual, n (\%) & $38(46,3)$ \\
\hline Infarto do miocárdio prévio, n (\%) & $17(20,7)$ \\
\hline Valor da creatinina basal, mg/dl (média \pm desvio padrão) & $1,08 \pm 0,31$ \\
\hline \multicolumn{2}{|l|}{ Localização do vaso culpado, n (\%) } \\
\hline Tronco da artéria coronária esquerda & 0 \\
\hline Artéria descendente anterior & $38(46,3)$ \\
\hline Artéria circunflexa & $11(13,4)$ \\
\hline Artéria coronária direita & $33(40,2)$ \\
\hline Diâmetro de referência do vaso proximal ao ponto de oclusão, mm (média \pm desvio padrão) & $2,80 \pm 0,6$ \\
\hline
\end{tabular}




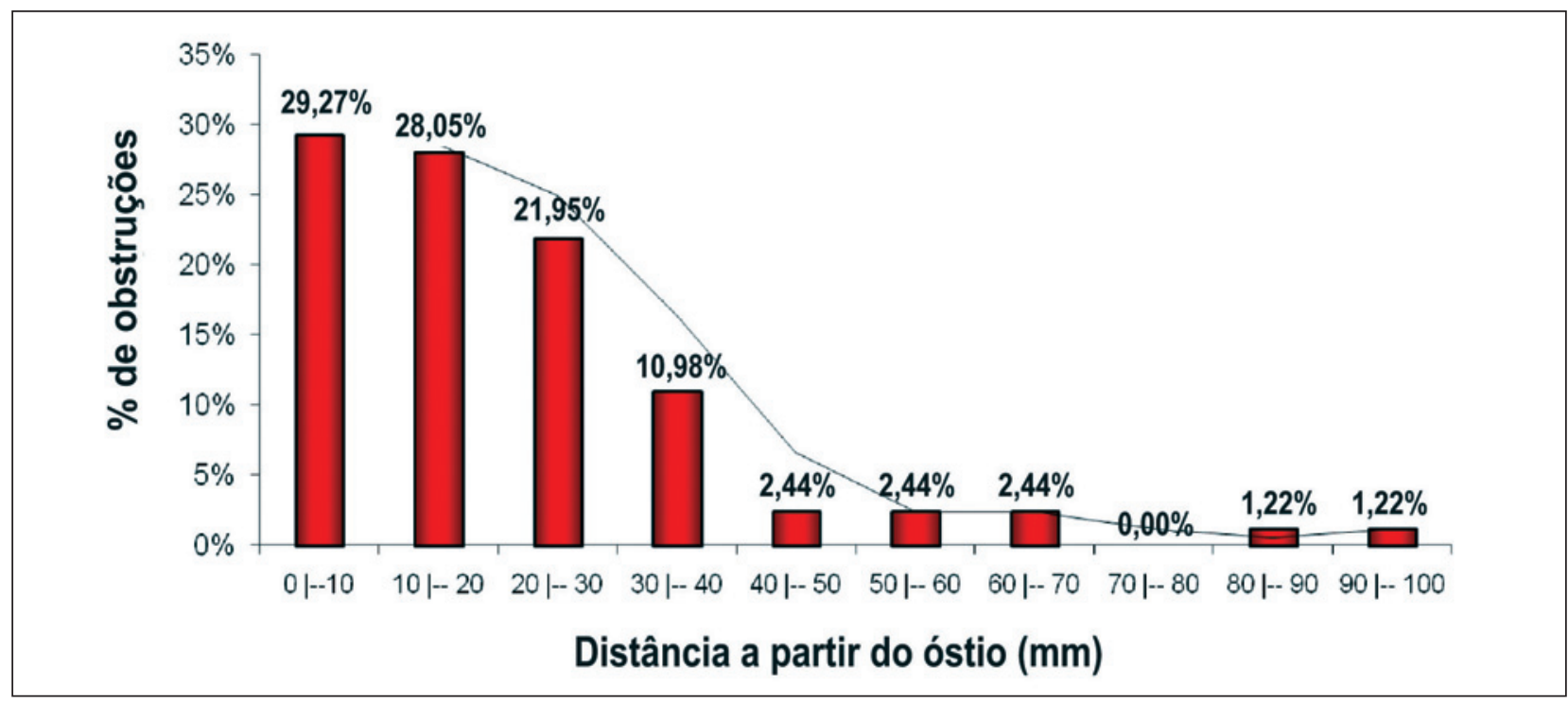

Figura 1 - Distribuição da ocorrência das oclusões trombóticas de artérias coronárias epicárdicas como uma função da distância do óstio coronário.

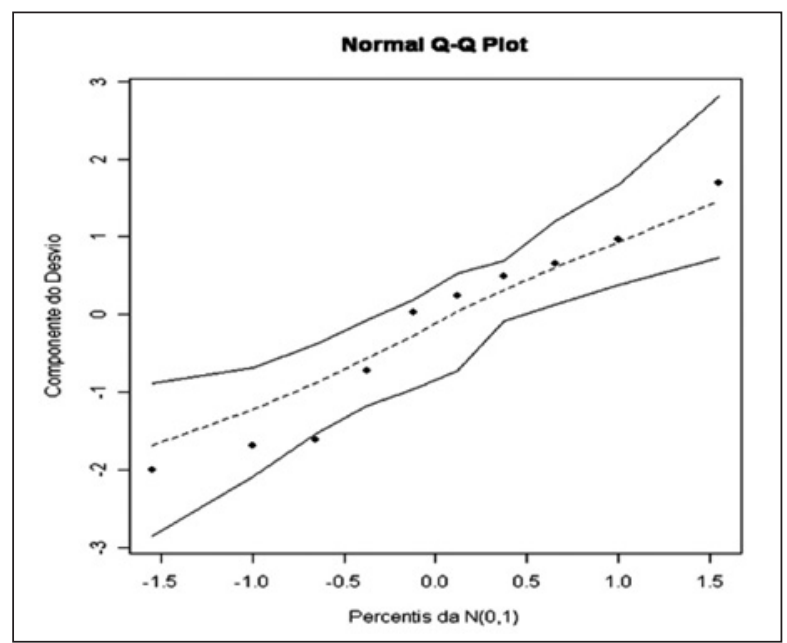

Figura 2 - Gráfico de envelope, indicando adequado ajuste do modelo de regressão aos dados avaliados.

$\mathrm{ca}^{26}$ e imagem nuclear ${ }^{27}$, estão em intensa investigação para a detecção desses tipos de placas.

Capacidade de identificar os pacientes de maior risco, presença e localização das lesões mais propensas a ruptura, e modificação de sua composição biológica podem trazer impacto importante na redução dos eventos cardiovasculares. Nesse sentido, o desenvolvimento de estratégias dedicadas ao tratamento das placas instáveis depende grandemente da capacidade de se reconhecer as lesões precursoras propensas a trombose.

Além de intervenções farmacológicas (aspirina, betabloqueadores, inibidores da enzima conversora de angiotensina e estatinas) e de modificações no estilo de vida (cessar o tabagismo, realização de atividades físicas, perda de peso, redução de estresse psicológico, etc.), tem sido sugerido que as placas instáveis podem ser seladas por meio de intervenção percutânea. A intervenção percutânea (com ou sem o uso de stents), ao promover ruptura física dessas placas, promoveria a substituição desse substrato vulnerável por tecido cicatricial (hiperplasia intimal), mais resistente a novas rupturas espontâneas ${ }^{28-30}$.

Identificar, por meio de um modelo angiográfico simples, os locais de maior concentração da ocorrência dessas placas pode auxiliar na otimização do uso racional dos recursos diagnósticos invasivos, mais custosos, bem como na decisão de se promover uma intervenção terapêutica invasiva.

\section{CONCLUSÕES}

As oclusões trombóticas agudas das artérias coronárias apresentam um padrão de distribuição nãouniforme, com maior propensão a ocorrer nos segmentos proximais do vaso epicárdico. O reconhecimento dessa característica pode ter implicações clínicas no uso racional e individualizado de recursos diagnósticos de imagem invasivos, bem como na indicação de intervenções coronárias percutâneas para modificação das características da placa aterosclerótica.

\section{CONFLITO DE INTERESSES}

Os autores declararam inexistência de conflito de interesses.

\section{REFERÊNCIAS BIBLIOGRÁFICAS}

1. Fuster V, Lewis A. Conner Memorial Lecture: mechanisms leading to myocardial infarction: insights from studies of vascular biology. Circulation. 1994;90(4):2126-46. 
Silva JFA, et al. Distribuição Espacial da Placa Vulnerável na Árvore Coronária: Avaliação Angiográfica de uma Coorte Consecutiva e Não-Selecionada de Pacientes Submetidos a Angioplastia Primária. Rev Bras Cardiol Invas. 2009;17(2):209-13.

2. Falk E, Shah PK, Fuster V. Coronary plaque disruption. Circulation. 1995;92(3):657-71.

3. Corti R, Farkouh ME, Badimon JJ. The vulnerable plaque and acute coronary syndromes. Am J Med. 2002;113(8):668-80.

4. Schoenhagen P, Tuzcu EM, Ellis SG. Plaque vulnerability, plaque rupture, and acute coronary syndromes: (multi)focal manifestation of a systemic disease process. Circulation. 2002;106(7):760-2.

5. Kolodgie FD, Burke AP, Farb A, Gold HK, Yuan J, Narula J, et al. The thin-cap fibroatheroma: a type of vulnerable plaque: the major precursor lesion to acute coronary syndromes. Curr Opin Cardiol. 2001;16(5):285-92.

6. Davies MJ, Richardson PD, Woolf N, Katz DR, Mann J. Risk of thrombosis in human atherosclerotic plaques: role of extracellular lipid, macrophage, and smooth muscle cell content. Br Heart J. 1993;69(5):377-81.

7. Kolodgie FD, Virmani R, Burke AP, Farb A, Weber DK, Kutys R, et al. Pathologic assessment of the vulnerable human coronary plaque. Heart. 2004;90(12):1385-91.

8. Wang JC, Normand SL, Mauri L, Kuntz RE. Coronary artery spatial distribution of acute myocardial infarction occlusions. Circulation. 2004;110(3):278-84.

9. el Fawal MA, Berg GA, Wheatley DJ, Harland WA. Sudden coronary death in Glasgow: nature and frequency of acute coronary lesions. Br Heart J. 1987;57(4):329-35.

10. Stefanadis C, Vavuranakis M, Toutouzas P. Vulnerable plaque: the challenge to identify and treat it. J Interv Cardiol. 2003; 16(3):273-80.

11. Thygesen K, Alpert JS, White HD, on behalf of the Joint ESC/ACCF/AHA/WHF Task Force for Redefinition of Myocardial Infarction. Universal definition of myocardial infarction. Circulation. 2007;116(22):2634-53.

12. Killip T, Fisher L, Mock $M$, for the CASS Investigators. National Heart, Lung, and Blood Institute Coronary Artery Surgery Study (CASS): a multicenter comparison of the effects of randomized medical and surgical treatment of mildly symptomatic patients with coronary artery disease, and a registry of consecutive patients undergoing coronary angiography. Circulation. 1981;63(Pt II, Suppl I):I-1-I-81.

13. Sheehan FH, Braunwald E, Canner P, Dodge HT, Gore J, Van Natta $P$, et al. The effect of intravenous thrombolytic therapy on left ventricular function: a report on tissue-type plasminogen activator and streptokinase from the Thrombolysis in Myocardial Infarction (TIMI Phase I) trial. Circulation. 1987;75(4):817-29.

14. Antman EM, Anbe DT, Armstrong PW, Bates ER, Green LA, Hand $M$, et al. ACC/AHA guidelines for the management of patients with ST-elevation myocardial infarction - executive summary: a report of the American College of Cardiology/ American Heart Association Task Force on Practice Guidelines (Writing Committee to Revise the 1999 Guidelines for the Management of Patients with Acute Myocardial Infarction). Circulation. 2004;110(5):588-636.

15. Burke AP, Farb A, Malcom GT, Liang YH, Smialek J, Virmani R. Coronary risk factors and plaque morphology in men with coronary disease who die suddenly. N Engl J Med. 1997;336(18):1276-82.

16. Virmani R, Kolodgie FD, Burke AP, Farb A, Schwartz SM.
Lessons from sudden coronary death: a comprehensive morphological classification scheme for atherosclerotic lesions. Arterioscler Thromb Vasc Biol. 2000;20(5):1262-75.

17. Schaar JA, Muller JE, Falk E, Virmani R, Fuster V, Serruys PW, et al. Terminology for high-risk and vulnerable coronary artery plaques. Report of a meeting on the vulnerable plaque, June 17 and 18, 2003, Santorini, Greece. Eur Heart J. 2004;25(12):1077-82.

18. Lee RT, Libby P. The unstable atheroma. Arterioscler Thromb Vasc Biol. 1997;17(10):1859-67.

19. Burke AP, Virmani R, Galis Z, Haudenschild CC, Muller JE. $34^{\text {th }}$ Bethesda Conference: Task Force \#2 - What is the pathologic basis for new atherosclerosis imaging techniques? J Am Coll Cardiol. 2003;41(11):1874-86.

20. Kolodgie FD, Narula J, Yuan C, Burke AP, Finn AV, Virmani R. Elimination of neoangiogenesis for plaque stabilization: is there a role for local drug therapy? J Am Coll Cardiol. 2007;49(21):2093-101.

21. Jang IK, Bouma BE, Kang DH, Park SJ, Park SW, Seung KB, et al. Visualization of coronary atherosclerotic plaques in patients using optical coherence tomography: comparison with intravascular ultrasound. J Am Coll Cardiol. 2002;39(4): 604-9.

22. Ambrose JA, Winters SL, Arora RR, Eng A, Riccio A, Gorlin $R$, et al. Angiographic evolution of coronary artery morphology in unstable angina. J Am Coll Cardiol. 1986;7(3):472-8.

23. Lerakis S, Synetos A, Toutouzas K, Vavuranakis M, Tsiamis E, Stefanadis C. Imaging of the vulnerable plaque: noninvasive and invasive techniques. Am J Med Sci. 2008;336(4):342-8.

24. Achenbach S, Moselewski F, Ropers D, Ferencik M, Hoffmann $U$, MacNeill B, et al. Detection of calcified and noncalcified coronary atherosclerotic plaque by contrast-enhanced, submilimiter multidetector spiral computed tomography: a segment-based comparison with intravascular ultrasound. Circulation. 2004;109(1):14-7.

25. Moselewski F, Ropers D, Pohle K, Hoffmann U, Ferencik M, Chan RC, et al. Comparison of measurement of cross-sectional coronary atherosclerotic plaque and vessel areas by 16 -slice multidetector computed tomography versus intravascular ultrasound. Am J Cardiol. 2004;94(10):1294-7.

26. Wilensky RL, Song HK, Ferrari VA. Role of magnetic resonance and intravascular magnetic resonance in the detection of vulnerable plaques. J Am Coll Cardiol. 2006;47 Suppl 8:C48-56.

27. Schäfers M, Riemann B, Kopka K, Breyholz HJ, Wagner S, Schäfers KP, et al. Scintigraphic imaging of matrix metalloproteinase activity in the arterial wall in vivo. Circulation. 2004;109(21):2554-9.

28. Meier B, Ramamurthy S. Plaque sealing by coronary angioplasty. Cathet Cardiovasc Diagn. 1995;36(4):295-7.

29. Kuntz RE. Importance of considering atherosclerotic progression when choosing a coronary revascularization strategy: the diabetes-percutaneous transluminal coronary angioplasty dilemma. Circulation. 1999;99(7):847-51.

30. Echeverri D, Purushothaman KR, Moreno PR. Reparação vascular após implante de stents não farmacológicos e farmacológicos em modelo experimental de fibroateroma de capa fina em coelhos. Rev Bras Cardiol Invas. 2008;16(4):474-81. 